\title{
Efeito Alelopático de Extratos Aquosos de Senna obtusifolia (L.) H. Irwin e Barneby
}

\author{
José Ricardo Barbosa da Silva ${ }^{1}$, Aldenir Feitosa dos Santos ${ }^{2}$ \\ ${ }^{1}$ Programa de Pós-Graduação em Biologia Vegetal - PPGBV, Departamento de Botânica, \\ Centro de Ciências Biológicas - CCB, Universidade Federal de Pernambuco - UFPE \\ ${ }^{2}$ Departamento de Química, Universidade Estadual de Alagoas - UNEAL
}

\begin{abstract}
RESUMO
Neste trabalho, estudou-se a germinação de sementes de Lycopersicum esculentum submetidas a extratos Senna obtusifolia. Foram realizados ensaios para análise da germinabilidade e velocidade de germinação. Os dados obtidos foram submetidos à ANOVA e ao teste de Tukey a $5 \%$ de probabilidade. Os extratos foram submetidos à prospecção de constituintes químicos. Tanto a análise da germinabilidade como a da velocidade de germinação demonstraram efeitos negativos consideráveis, sendo que os extratos de folha foram os que mais evidenciaram resultados significativos. Na prospecção de constituintes químicos, foi caracterizada a presença de variados grupos de metabólitos secundários nos três tipos de extrato.
\end{abstract}

Palavras-chave: alelopatia, aleloquímico, mata-pasto.

\section{Allelopathic Potential of Aqueous Extracts of Senna obtusifolia (L.) H. Irwin and Barneby}

\begin{abstract}
The aim of this work was to study the seeds germination of Lycopersicum sculentum when subjected to extracts of Senna obtusifolia. Bioassays were performed in order to analyze germinability and germination speed rate. The data obtained in the bioassays were subjected to ANOVA and test of Tukey at 5\% probability. The extracts were subjected to chemical prospecting. Both germinability and germination speed rate analyses showed negative effects and the leaves extracts presented the most significant results. The presence of various groups of secondary metabolites was characterized in the chemical prospecting of three types of extracts.
\end{abstract}

Keywords: allelopathy, allelochemical, obtusifolia. 


\section{INTRODUÇÃO}

A alelopatia é a interferência positiva ou negativa promovida por uma espécie vegetal quando esta sintetiza e disponibiliza no meio metabólitos secundários que possuem a capacidade de influenciar direta ou indiretamente no estabelecimento de outros organismos (Ferreira, 2004).

Esse tipo de interferência tem sido muito estudada e o avanço dessa linha de pesquisa devese a três principais fatores: os efeitos alelopáticos são a chave para o entendimento de distintas relações ecológicas como em alguns casos de sucessão ecológica (Ferreira, 2004) e de invasão biológica (Perdomo \& Magalhães, 2007), por exemplo; pelo fato de que o isolamento e identificação de aleloquímicos possibilitam a descoberta de herbicidas naturais (Haig et al., 2005; Cornes, 2005); pelo motivo ainda de possibilitar descobertas de defensivos agrícolas naturais contra doenças, parasitas, herbivoria, etc. (Halbrendt, 1996).

Senna obtusifolia (L.) H. Irwin e Barneby (família Fabaceae, subfamília Caesalpinioideae), conhecida popularmente como fedegoso, fedegoso-branco, vagem-foice, mata-pasto-liso ou simplesmente matapasto, é uma planta daninha que tem assumido grande importância como infestante (Ávila et al., 2000), tornando-se assim um problema para agricultores de soja do Brasil (Mello et al., 2001). É também uma das espécies mais frequentes, infestando solos de cultivo intensivo, bem como pastagens, pomares e terrenos baldios (Mello et al., 2003). Por estes motivos Senna obtusifolia é considerada hoje uma espécie invasora muito agressiva que tem sido motivo de vários estudos visando seu controle (Ávila et al., 2000; Mello et al., 2003).

Diante da inexistência de trabalhos similares sobre Senna obtusifolia e alelopatia e da forte relação encontrada na literatura entre invasão e alelopatia (Barbosa, et al., 2008, Inderjit et al., 2008; Perdomo \& Magalhães, 2007; Souza, 2006), este trabalho teve como principal objetivo testar a hipótese de que Senna obtusifolia possui a capacidade de interferir negativamente na germinação de sementes de outras espécies vegetais através de alelopatia, demonstrando isso por extratos aquosos testados em laboratório.

\section{MATERIAL E MÉTODOS}

Os experimentos foram conduzidos nos laboratórios multidisciplinares de Biologia e de Química do Campus I - Arapiraca da Universidade Estadual de Alagoas - UNEAL durante o período de 28 de agosto a 15 de dezembro de 2006. O trabalho foi dividido em três etapas distintas.

\subsection{Obtenção de extratos}

Foram coletadas amostras de raízes, caules e folhas de Senna obtusifolia no sítio Massaranduba na zona rural de Arapiraca-AL em 28 de agosto de 2006. O material coletado foi selecionado considerando a morfologia, inexistência de parasitas, tamanho e aparência geral, em seguida as amostras foram levadas ao laboratório de Química da UNEAL onde foram lavadas e, em seguida, trituradas separadamente em liquidificador doméstico. Após trituração, foram preparadas soluções aquosas das amostras vegetais na concentração de $0,2 \mathrm{~g} / \mathrm{mL}$ (30 g de cada região do vegetal em $150 \mathrm{~mL}$ de água destilada). O tempo de extração foi de 96 horas sendo realizado sob refrigeração a uma temperatura constante de $10^{\circ} \mathrm{C}$ para evitar a proliferação de fungos. Após o período de extração, o material foi submetido à filtração simples. Os extratos obtidos (de concentração igual a $0,2 \mathrm{~g} / \mathrm{mL}$ ou $20 \% \mathrm{p} / \mathrm{v}$ ) foram denominados de extratos brutos (ou 100\%), a partir dos quais foram obtidos os extratos diluídos em água destilada no percentual de 70 e $30 \%$ de cada extrato bruto. Ao final da etapa de preparação de extratos, foram obtidos três extratos das raízes, três extratos de caules e três extratos das folhas. Cada extrato teve seu $\mathrm{pH}$ medido com papel de tornassol (escala de 1 a 14) e, como não foi evidenciada diferença significativa quando comparado ao $\mathrm{pH}$ da água destilada, $\mathrm{o} \mathrm{pH}$ dos extratos não necessitou de correção.

\subsection{Ensaios para germinação}

Para os ensaios foram utilizadas placas de Petri esterilizadas por uma hora a $100{ }^{\circ} \mathrm{C}$ em estufa. As placas foram forradas com duas folhas de papel de filtro também esterilizadas, nas quais foram colocadas 25 sementes de Lycopersicum esculentum por placa, e, em seguida, umedecidas com $5 \mathrm{~mL}$ de extrato (ou água destilada nas placas com o controle); 
logo após as placas foram tampadas e vedadas com filme de plástico (Ferreira, 2004). As placas foram então dispostas em delineamento inteiramente casualizado através de sorteio (Ferreira, 2004) dentro de uma caixa de madeira medindo $40 \mathrm{~cm}$ de largura, $120 \mathrm{~cm}$ de comprimento e $50 \mathrm{~cm}$ de altura com isolamento da luz, a qual ficou em bancada no laboratório de Química da UNEAL a $25{ }^{\circ} \mathrm{C}$ durante todo o experimento. Para cada tipo de extrato, foram utilizadas quatro repetições, totalizando 100 sementes por extrato e 40 placas ao todo. Foram realizadas observações diárias sempre às 20 horas durante 96 horas sob iluminação somente de luz verde difusa. Foram consideradas germinadas as sementes que apresentaram protusão radicular a partir de $2 \mathrm{~mm}$ de comprimento (Brasil, 1992), as quais foram contabilizadas para as análises de germinabilidade $(\% \mathrm{G})$ e velocidade de germinação (VG). A germinabilidade foi considerada como o índice de sementes germinadas de um total em um determinado tempo (Ferreira \& Áquila, 2000) e a velocidade de germinação foi calculada segundo a fórmula proposta por Edmond \& Daprala (1985). Subsequentemente, os resultados verificados tiveram suas médias comparadas pela análise da variância e sua significância pelo teste de Tukey a $5 \%$ de probabilidade com auxílio do software Assistat 7.5 Beta.

\subsection{Screening fitoquímico}

Para a realização da etapa de triagem fitoquímica, tomou-se como base a metodologia proposta por Matos (1988), a qual foi trabalhada a fim de realizar prospecção dos seguintes aleloquímicos: fenóis, taninos pirogálicos, taninos flobafênicos, antocianina, antocianidina, flavonas, flavonóis, xantonas, chalconas, auronas, flavononóis, leucoantocianidinas, catequinas, flavononas, flavonóis, xantonas, esteroides, triterpenoides e saponinas. De cada extrato, foram separadas cinco amostras de $3,5 \mathrm{~mL}$ e uma amostra de $10 \mathrm{~mL}$ em tubos de ensaio, as quais sofreram processos de acidificação ou alcalinização, entre outros. A indicação da formação de espuma e mudança da cor foram as características para a determinação da presença ou ausência de metabólitos secundários nas amostras. Foram realizados controles negativos com água destilada para cada teste. A comparação das cores foi feita com auxílio de um padrão de cores fornecido pela Suvinil ${ }^{\circ}$.

Para a determinação de fenóis, taninos pirogálicos e taninos flobafênicos em uma amostra, foram colocadas três gotas de solução alcoólica de $\mathrm{FeCl}_{3}$, após agitação foi observada a ocorrência de variação de cor ou formação de precipitado abundante escuro. A coloração entre o azul e o vermelho seria indicativa de fenóis, precipitado escuro de tonalidade azul seria indicativa da presença de taninos pirogálicos (taninos hidrolisáveis) e verde da presença de taninos flobafênicos (taninos condensados ou catéquicos).

Para a identificação de antocianina e antocianidina; flavonas, flavonóis e xantonas; chalconas e auronas; e flavononóis, uma amostra foi acidificada com $\mathrm{HCl}$ a $\mathrm{pH} 3,0$, uma amostra foi alcalinizada a pH 8,5 e outra alcalinizada a pH 11,0, ambas através da adição de $\mathrm{NaOH}$. A cor vermelha na primeira amostra, acompanhada das cores lilás na segunda e azul-púrpura na terceira, seria indicativa da presença de antocianinas e antocianidina. A cor amarela na terceira amostra, sem mudança de cor nas demais, indicaria a presença de flavonas, flavonóis e xantonas. A cor vermelha na primeira amostra, seguida de vermelho-púrpura na terceira, indicaria a presença de chalconas e auronas. A cor vermelho-laranja na terceira amostra, sem alteração na cor das demais amostras, indicaria a presença de flavononóis.

Para testar a presença de leucoantocianidinas, catequinas e flavononas, uma amostra foi acidificado com $\mathrm{HCl}$ até $\mathrm{pH}$ 2,0 e outra amostra alcalinizada com $\mathrm{NaOH}$ a pH 11,0. Ambas as amostras foram aquecidas em banho-maria a $100{ }^{\circ} \mathrm{C}$ por 3 minutos. Após o aquecimento, a cor vermelha na amostra acidificada indicaria a presença de leucoantocianidinas, a cor pardo-amarela na mesma amostra seria indicativa da presença de catequinas e a cor vermelho-alaranjada seria indicativa da presença de flavonononas.

Para os testes de esteroides e triterpenos, as amostras de $10 \mathrm{~mL}$ foram evaporadas e os resíduos extraídos com $2 \mathrm{~mL}$ de clorofórmio por três vezes. Foi adicionado às amostras $1 \mathrm{~mL}$ de anidrido acético e $0,5 \mathrm{~mL}$ de $\mathrm{H}_{2} \mathrm{SO}_{4}$, após agitação, a coloração azul evanescente seguida de verde permanente indicaria a presença de esteroides livres. A coloração parda 
até vermelha indicaria triterpenoides pentacíclicos livres.

Para os testes de saponinas, o resíduo insolúvel em clorofórmio, separado na operação anterior, foi ressuspendido em $8 \mathrm{~mL}$ de água destilada e a solução foi filtrada para um tubo de ensaio. Agitouse o tubo com a solução por 3 minutos e observou-se a formação de espuma, a qual, sendo persistente e abundante (colarinho), seria indicativa da presença de saponinas (esteroides saponínicos).

\section{RESULTADOS E DISCUSSÃO}

$\mathrm{Na}$ avaliação da germinabilidade, observouse, conforme tabela 1 , que nas primeiras 48 horas os extratos de Folha a 100, 70 e $30 \%$ foram os que apresentaram maior inibição de germinação seguidos do estrato de Raiz a 100\%. Em 72 horas, os extratos de Folhas a 70 e $100 \%$ foram os que apresentaram um maior índice de inibição de germinação, seguidos do extrato de Raiz a 100\%. Em 96 horas, o único extrato que apresentou uma diferença significativa foi o de Folhas a $100 \%$.

De acordo com a Tabela 1, pode-se observar que há uma variada ação dos extratos na inibição da germinação e que esta apresenta uma maior sensibilidade aos efeitos alelopáticos nas horas iniciais da germinação. Pode-se notar também que o extrato de Folha a $100 \%$ é o único que durante todo o período de observações consegue manter uma diferença significativa com relação ao índice de germinação do controle.
Analisando o experimento como um todo durante as 96 horas, pode-se notar que a germinação ocorrida nos ensaios umedecidos com água destilada apresentou uma progressão acentuada na germinação entre 48 e 72 horas e que durante o período compreendido entre 72 e 96 horas houve uma relativa e visível estabilidade na germinação, o que propõe um pico na germinação de sementes de tomate com 72 horas após o contato com a água, conforme relata Ferreira (2004). A Figura 1 demonstra essa condição na germinação dos ensaios controle e aponta ainda que os ensaios com o extrato de Folhas a 100\% foram os únicos que apresentaram um comportamento exatamente inverso ao do controle, com uma germinação relativamente moderada e estável durante o período de 48 a 72 horas e com um visível crescimento no índice de germinação entre 72 e 96 horas. Os demais ensaios apresentaram um comportamento semelhante ao do controle em pelo menos um dos intervalos citados.

Outro fato é que a influência negativa no percentual de sementes germinadas é diretamente proporcional às concentrações dos extratos em cada tipo de extrato. Segundo Inderjit (2008), os aleloquímicos podem agir na germinação de acordo com aspectos qualitativos e/ou quantitativos, dessa maneira sugere-se que os efeitos evidenciados pelos extratos de Folhas foram mais eficientes na concentração de $100 \%$ por causa das concentrações de metabólitos que ocasionaram tais efeitos. Por outro lado, a maior eficiência dos extratos de Folhas em relação aos de Caules e de Raízes pode ter

Tabela 1. Germinabilidade (\%) de sementes de tomate submetidas a diferentes extratos aquosos de S. obtusifolia observadas em 48, 72 e 96 horas.

Table 1. Germination of tomato seeds exposed to different aqueous extracts of S. obtusifolia observed in 48,72 and 96 hours.

\begin{tabular}{|c|c|c|c|c|c|c|c|c|c|c|}
\hline \multirow{3}{*}{ Observações } & \multicolumn{10}{|c|}{ Tipo de extrato } \\
\hline & \multirow{2}{*}{$\begin{array}{c}\text { Controle } \\
(-)\end{array}$} & \multicolumn{3}{|c|}{ Folhas } & \multicolumn{3}{|c|}{ Caule } & \multicolumn{3}{|c|}{ Raiz } \\
\hline & & $30 \%$ & $70 \%$ & $100 \%$ & $30 \%$ & $70 \%$ & $100 \%$ & $30 \%$ & $70 \%$ & $100 \%$ \\
\hline 48 horas & $52^{\mathrm{a}}$ & $2^{\mathrm{e}}$ & $0^{\mathrm{e}}$ & $0^{\mathrm{e}}$ & $44^{\mathrm{ab}}$ & $36^{\mathrm{b}}$ & $23^{c}$ & $34^{\mathrm{b}}$ & $13^{\mathrm{cd}}$ & $3^{\text {de }}$ \\
\hline 72 horas & $85^{\mathrm{a}}$ & $64^{\mathrm{ab}}$ & $36^{\mathrm{cd}}$ & $12^{\mathrm{d}}$ & $82^{\mathrm{a}}$ & $82^{\mathrm{a}}$ & $79^{\mathrm{a}}$ & $78^{\mathrm{a}}$ & $67^{\mathrm{ab}}$ & $53^{\mathrm{bc}}$ \\
\hline 96 horas & $90^{\mathrm{a}}$ & $93^{\mathrm{a}}$ & $86^{\mathrm{a}}$ & $63^{\mathrm{b}}$ & $93^{\mathrm{a}}$ & $89^{\mathrm{a}}$ & $87^{\mathrm{a}}$ & $89^{\mathrm{a}}$ & $78^{\mathrm{ab}}$ & $85^{\mathrm{a}}$ \\
\hline
\end{tabular}

Médias dentro da mesma linha, que comparam extratos diferentes em períodos de observação iguais, seguidas das mesmas letras não diferem significativamente entre si, pelo teste de Tukey, ao nível de 5\% de probabilidade. Para observações em 48 horas: MG = 5,17500; $\mathrm{CV} \%=21,81950 ; \mathrm{GL}=39 ; \mathrm{F}=76,6471^{*}$. Para observações em 72 horas: $\mathrm{MG}=15,95000 ; \mathrm{CV} \%=15,61116 ; \mathrm{GL}=39 ; \mathrm{F}=$ $22,9731^{*}$. Para observações em 96 horas: $\mathrm{MG}=21,32500 ; \mathrm{CV} \%=9,91990 ; \mathrm{GL}=39 ; \mathrm{F}=4,4823^{*}$. ${ }^{*}$ Estatística do teste $\mathrm{F}$ significativo ao nível de $1 \%$ de probabilidade (p-valor $<0.01$ ). 


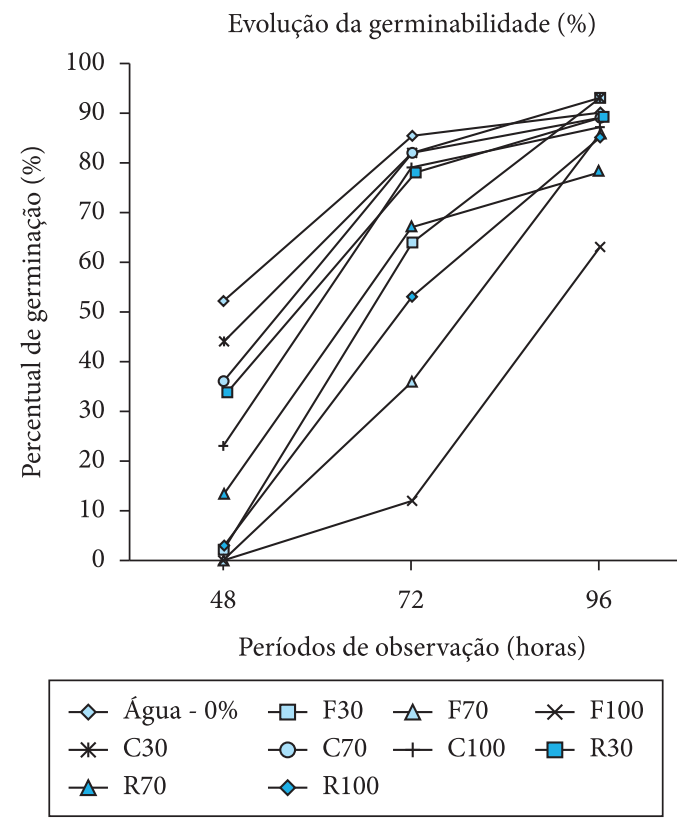

Figura 1. Progressão da germinabilidade de sementes de tomate submetidas a diferentes extratos aquosos de S. obtusifolia observadas entre 48 e 96 horas. A letra na legenda corresponde à parte vegetal $(\mathrm{F}=$ folha, $\mathrm{C}=$ caule e $\mathrm{R}=$ raiz) e os números correspondem à concentração do extrato.

Figure 1. Germination of tomato seeds exposed to different aqueous extracts of S. obtusifolia. The lyrics compare different extracts in equal periods of observation. Same letters in the same periods of observation did not differ significantly by Tukey test at $5 \%$ probability.

ocorrido tanto por diferenças entre os metabólitos secundários como também pelas suas concentrações.

$\mathrm{Na}$ análise da velocidade de germinação, conforme a Tabela 2, durante as primeiras 48 horas as médias de velocidade de germinação dos extratos de Folhas a 70 e a $100 \%$ apresentaram velocidade nula, já os demais extratos tiveram suas médias de velocidade de germinação semelhante ao do controle. Durante a observação realizada em 72 horas, notou-se que apenas o extrato de Folha a $100 \%$ reduziu consideravelmente a velocidade de germinação. Já com 96 horas, os únicos extratos que não demonstraram ação redutora estatisticamente considerável na velocidade de germinação foram os extratos de Caule a 30\%, Caule a 70\% e Raiz a 30\%. Os extratos de Folha a $70 \%$ e Folha a $100 \%$ foram os
Tabela 2. Velocidade de germinação $\left(\operatorname{dias}^{-1}\right)$ de sementes de tomate submetidas a diferentes extratos aquosos de S. obtusifolia.

Table 2. Speed of germination of tomato seeds exposed to different aqueous extracts of S. obtusifolia.

\begin{tabular}{|lccc|}
\hline \multicolumn{4}{c|}{ Velocidade de germinação em di ${ }^{\text {as }} \mathbf{1}$} \\
\hline $\begin{array}{c}\text { Tipo de } \\
\text { extrato }\end{array}$ & $\mathbf{4 8}$ horas & $\mathbf{7 6}$ horas & $\mathbf{9 6}$ horas \\
\hline Controle (-) & $0,500^{\mathrm{a}}$ & $0,418^{\mathrm{a}}$ & $0,404^{\mathrm{a}}$ \\
\hline Folha $30 \%$ & $0,250^{\mathrm{ab}}$ & $0,336^{\mathrm{ab}}$ & $0,303^{\mathrm{de}}$ \\
\hline Folha 70\% & $0^{\mathrm{b}}$ & $0,333^{\mathrm{ab}}$ & $0,278^{\mathrm{e}}$ \\
\hline Folha $100 \%$ & $0^{\mathrm{b}}$ & $0,250^{\mathrm{b}}$ & $0,260^{\mathrm{e}}$ \\
\hline Caule 30\% & $0,500^{\mathrm{a}}$ & $0,406^{\mathrm{a}}$ & $0,378^{\mathrm{ab}}$ \\
\hline Caule 70\% & $0,500^{\mathrm{a}}$ & $0,390^{\mathrm{a}}$ & $0,374^{\mathrm{ab}}$ \\
\hline Caule $100 \%$ & $0,500^{\mathrm{a}}$ & $0,370^{\mathrm{ab}}$ & $0,353^{\mathrm{bc}}$ \\
\hline Raiz 30\% & $0,500^{\mathrm{a}}$ & $0,389^{\mathrm{a}}$ & $0,366^{\mathrm{abc}}$ \\
\hline Raiz 70\% & $0,500^{\mathrm{a}}$ & $0,356^{\mathrm{ab}}$ & $0,336^{\mathrm{bcd}}$ \\
\hline Raiz $100 \%$ & $0,250^{\mathrm{ab}}$ & $0,340^{\mathrm{ab}}$ & $0,299^{\mathrm{cd}}$ \\
\hline
\end{tabular}

Médias dentro da mesma coluna, que comparam extratos diferentes em períodos de observação iguais, seguidas das mesmas letras não diferem significativamente entre si, pelo teste de Tukey, ao nível de 5\% de probabilidade. Para observações em 48 horas: $\mathrm{MG}=0,35000$; $\mathrm{CV} \%=36,88556$; $\mathrm{GL}=39 ; \mathrm{F}=10.6667^{*}$. Para observações em 72 horas: MG $=0,35825 ; \mathrm{CV} \%=15,35029 ; \mathrm{GL}=39 ; \mathrm{F}=3.0294^{*}$. Para observações em 96 horas: $\mathrm{MG}=0,33250 ; \mathrm{CV} \%=5,66660$; GL $=39 ; \mathrm{F}=23.8811^{*} .{ }^{*}$ Estatística do teste $\mathrm{F}$ significativo ao nível de $1 \%$ de probabilidade ( $\mathrm{p}$-valor $<0.01$ ).

que mais reduziram a velocidade de germinação no período.

Assim como na análise da germinabilidade, o extrato de Folha a $100 \%$ foi aquele que mais influenciou as sementes de tomate quanto à velocidade de germinação (Tabela 2). Durante as três observações realizadas, o extrato de Folha a 100\% se mostrou o de maior relevância em todas. Porém, a velocidade de germinação foi muito mais sensível aos efeitos alelopáticos que a germinabilidade, a qual, na última observação, foi influenciada significativamente somente pelo extrato de Folha a $100 \%$, enquanto que, ao fim do experimento, a velocidade de germinação foi influenciada pelos extratos de Folha a 30, 70 e 100\%, pelo de Caule a $100 \%$ e pelos de Raiz a 70 e a $100 \%$.

Nota-se ainda que, em cada tipo de extrato, sempre os de maior concentração são os mais eficiente na redução da velocidade de germinação. Como foi 
discutido na análise da germinabilidade, este fato pode ocorrer por causa das diferentes concentrações de metabólitos secundários que promoveram tal efeito. Porém, a maior eficiência evidenciada nos extratos de Folhas em relação aos de Caules e Raízes pode ter ocorrido não só por uma diferença quantitativa das concentrações de aleloquímicos, mas em conjunto ou não com diferenças qualitativas de tais metabólitos.

A Figura 2 mostra a evolução da velocidade de germinação das sementes de tomate expostas aos extratos e deixa evidente a diferença do comportamento da velocidade de germinação das sementes submetidas aos extratos de Folha a 70 e $100 \%$, quando comparados com os demais, os quais foram os únicos que mostraram um comportamento exatamente inverso aos demais extratos.

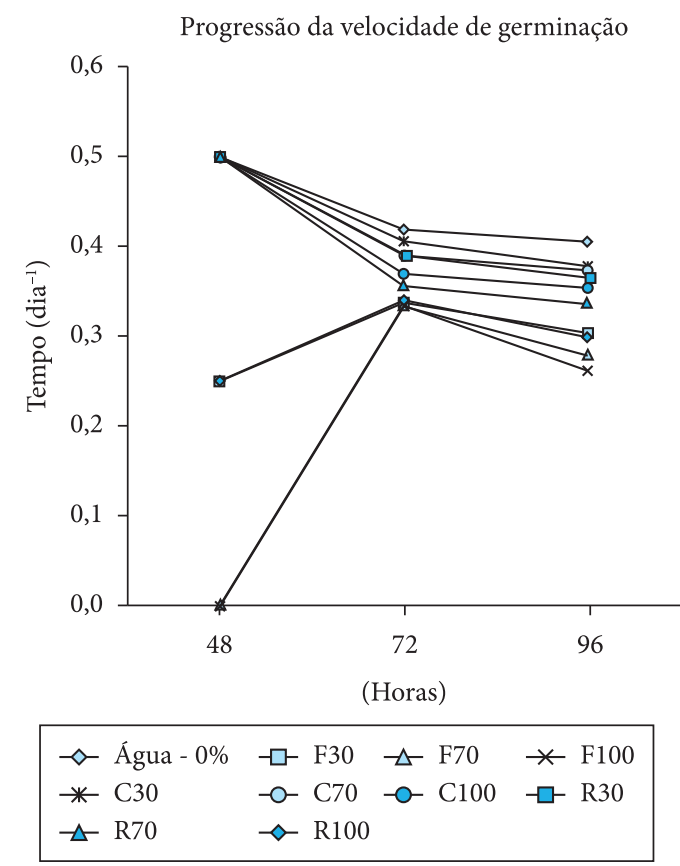

Figura 2. Progressão da velocidade de germinação em sementes de tomate submetidas a diferentes extratos aquosos de S. obtusifolia observadas entre 48 e 96 horas. As letras na legenda correspondem à parte vegetal ( $\mathrm{F}=$ folha, $\mathrm{C}$ = caule e $\mathrm{R}=$ raiz) e os números correspondem à concentração do extrato.

Figure 2. Progression of the germination of tomato seeds exposed to different aqueous extracts of $S$. obtusifolia observed between 48 and 96 hours. The letter in the legend corresponds to the plant ( $\mathrm{F}=$ leaf, stem and $\mathrm{C}=\mathrm{R}=$ root) and the numbers correspond to the concentration of the extract.
Segundo os testes fitoquímicos, nos extratos de Folhas e Caules, foi identificada a presença de taninos flobafênicos, esteroides e saponinas. Nos testes nos extratos de raízes, identificou-se a presença de fenóis, flavononóis, triterpenoides e saponinas. A Tabela 3 mostra tais resultados com a presença ou ausência dos aleloquímicos testados.

Nos extratos de folhas e de caules, foram identificados os mesmos aleloquímicos. Já os extratos de raízes além das saponinas, que também estavam presentes nos outros dois tipos de extratos, também apresentaram três grupos de metabólitos diferentes. Diante da similaridade química entre grupos de metabólitos encontrados nos extratos de folhas e nos demais extratos, pode-se sugerir que a maior eficiência do extrato de Folhas tenha sido causada por diferenças específicas entre compostos dentro dos grupos de metabólitos estudados, por uma diferença quantitativa de tais metabólitos ou pela soma desses dois fatores.

Tabela 3. Prospecção dos constituintes químicos de extratos aquosos de folha, caule e raiz de S. obtusifolia.

Table 3. Prospecting of the chemical constituents of aqueous extracts of leaf, stem and root of S. obtusifolia.

\begin{tabular}{|c|c|c|c|}
\hline \multirow{2}{*}{ Aleloquímico } & \multicolumn{3}{|c|}{ Tipo de extrato } \\
\hline & Folha & Caule & Raiz \\
\hline Fenóis & - & - & * \\
\hline Taninos pirogálicos & - & - & - \\
\hline Taninos flobafênicos & * & * & - \\
\hline Antocianina e antocianidina & - & - & - \\
\hline Flavonas, flavonóis e xantonas & - & - & - \\
\hline Chalconas e auronas & - & - & - \\
\hline Flavononóis & - & - & * \\
\hline Leucoanto e cianidinas & - & - & - \\
\hline Catequinas & - & - & - \\
\hline Flavononas & - & - & - \\
\hline $\begin{array}{l}\text { Flavonóis, flavononas, } \\
\text { flavonóis e xantonas }\end{array}$ & - & - & - \\
\hline Esteroides & * & * & - \\
\hline Triterpenoides & - & - & * \\
\hline Saponinas & * & * & * \\
\hline
\end{tabular}

${ }^{*}$ Indica presença e - indica ausência. 


\section{CONCLUSÕES}

Os extratos aquosos de Senna obtusifolia causaram inibição na germinabilidade e redução da velocidade de germinação de sementes de Lycopersicum esculentum, ocasionando assim efeitos alelopáticos em ensaios laboratoriais.

Os extratos de folha foram os que mais demonstraram eficiência na inibição da germinabilidade e redução da velocidade de germinação, sendo que o extrato de folha a $100 \%$ foi o que apresentou maior eficiência em tais efeitos.

$\mathrm{Na}$ prospecção química foram evidenciados classes de metabólitos secundários diferentes que podem ser os responsáveis pelos efeitos biológicos constatados.

Levando-se em consideração que o solvente utilizado foi água e que as concentrações dos extratos foram semelhantes às usuais em trabalhos sobre alelopatia, os resultados obtidos sugerem que Senna obtusifolia é uma espécie interessante para a realização de outros trabalhos sobre alelopatia, principalmente visando a obtenção de extratos herbicidas.

\section{AGRADECIMENTOS}

À Universidade Estadual de Alagoas - UNEAL pela realização dos experimentos em suas instalações.

À Coordenação de Aperfeiçoamento de Pessoal de Nível Superior - CAPES pela bolsa de mestrado.

À Universidade Federal de Pernambuco - UFPE pela colaboração com os experimentos.

Ao Centro de Estudos Superiores de Maceió - CESMAC pela colaboração financeira para a realização do trabalho.

\section{STATUS DA SUBMISSÃO}

Recebido: 26/08/2009
AUTOR(ES) PARA CORRESPONDÊNCIA

\section{José Ricardo Barbosa da Silva}

Programa de Pós-Graduação em Biologia Vegetal, Departamento de Botânica, Centro de Ciências Biológicas - CCB, Universidade Federal de Pernambuco - UFPE, Av. Professor Moraes Rêgo, s/n, Cidade Universitária, CEP 50670-901

Recife, PE, Brasil

e-mail: ricardosilva_bio@hotmail.com.

\section{REFERÊNCIAS}

Ávila ZR, Mello SCM, Ribeiro ZMA, Fontes EMG. Produção de Inóculo de Alternaria Cassiae. Revista PAB Pesquisa Agropecuária Brasileira 2000; 35:533-541.

Barbosa EG, Pivello VR, Meirelles ST. Allelopathic Evidence in Brachiaria decumbens and its Potential to Invade the Brazilian Cerrados. Brazilian archives of Biology and Technology 2008; 5:825-831.

Brasil. Ministério da Agricultura e Reforma Agrária. Regras para análise de sementes. Brasília; 1992.

Cornes D. Callisto: a very successful maize herbicide inspired by allelochemistry. In: Fourth World Congress on Allelopathy. 2005; Wagga Wagga, NSW Australia. Available from: http://www.regional.org.au/au/ allelopathy/2005/2/7/2636_cornesd.htm.

Edmond JB, Drapala WJ. The effects of temperature, sand, soil, and acetone on germination of okra seeds. Proceedings of the American Society for Horticultural Sciencev 1958; 71:428-434.

Ferreira AG, Aquila MEA. Alelopatia: uma área emergente da ecofisiologia. Revista Brasileira de Fisiologia Vegetal 2000; 12:175-204.

Ferreira AG. Interferência: competição e alelopatia. In: Ferreira AG, Borghetti F, organizadores. Germinação do básico ao aplicado. Porto Alegre: Artimed; 2004. p. 207-222.

Haig T, Pratley J, An M, Haig T, Hildebrand S. Using allelopathy to search for new natural herbicides from plants. In: Fourth World Congress on Allelopathy. 2005; Wagga Wagga, NSW Australia. Available from: http://. regional.org.au/au/allelopathy/2005/2/7/2749_haigtj. htm.

Halbrendt JM. Allelopathy in the Management of PlantParasitic Nematodes. Journal of Nematology, 1996; 28:8-14

Inderjit, Seasted TR, Callaway RM, Pollock JL, Kaur J. Allelopathy and plant invasions: traditional, congeneric, and bio-geographical approaches. Biological Invasions 2008; 10:875-890. 
Matos FJA. Introdução à fitoquímica experimental. Fortaleza: EUFC; 1988.

Mello SCM, Ávila ZR, Estelles RS. Efeitos da idade da planta, concentração do inoculo e período úmido no controle de Senna obtusifolia por Alternaria cassiae. EMBRAPA; 2003. Comunicado técnico, 84.

Mello SCM, Ribeiro ZMA, Souza GR, Tigano M, Nachtigal GF, Fontes EMG. Padrões Isoenzimáticos e Morfologia de Isolados de Alternaria spp. Patogênicos a Senna obtusifolia. Fitopatologia Brasileira 2001; 26:667-669.

Perdomo M, Magalhães LMS. Ação alelopática da jaqueira (Artocarpus heterophyllus) em laboratório. Revista Floresta e Ambiente 2007; 14:52-55.

Souza APSF. Potencialidades alelopáticas envolvendo gramíneas e leguminosas forrageiras e plantas invasoras de pastagens. Pesquisa Agropecuária Brasileira 1997; 32:165-170. 